type which had both breast cancer and an interstitial-cell tumour of the testis. It is perhaps not surprising that a syndrome associated with progressive testicular atrophy and hyperplasia of Leydig cells should be associated with an increased risk of tumours derived from the latter element. This is the regular sequence of events in cadmium poisoning in rats. ${ }^{2}$ Perhaps the surprise is that the association has not been more obvious previously.

A disturbing finding from J. C. Heath in collaboration with others is that detritus from two vitallium (a cobalt-chromiummolybdenum alloy) articulatory surfaces rubbed together mechanically in Ringer's solution gave rise to a rhabdomyosarcoma after intramuscular injection into a rat. Also disturbing is the finding by J. Swinney and R. O. K. Schade that 80 out of 100 biopsy specimens of mucosa taken at some distance from transitional-cell carcinomas of the bladder showed changes they interpreted as precancerous. It is not clear how many of the 100 patients had a history of exposure to a known bladder carcinogen, but if such a history were uncommon and if the findings can be confirmed they could be taken to suggest that environmental carcinogens acting on the whole bladder mucosa are implicated in the causation of the majority of bladder cancers.

\section{A New Sign?}

Interpretation of the symptoms and signs of biliary disease may be difficult and inexact, and certain popular fallacies exist. One attributes flatulent dyspepsia to gall stones, whereas the facts show that gall stones and gall-bladder disease are not always present and that the symptoms are more nearly related to underlying patterns of abnormal contractility in the stomach and duodenum. Another fallacy concerns biliary colic; the pain is often believed to be sited over the position of the gall-bladder in the right hypochondrium, whereas it is usually felt across the upper abdomen, sometimes even starting in the left hypochondrium. The embryological development of the biliary system is from the foregut, which is a midline structure and symmetrically innervated.

If cholecystitis follows the colic the pain and tenderness will be felt in the right hypochondrium. This symptom sequence may be compared to that in acute appendicitis, where the central colicky abdominal pain (midgut) is followed by the pain and tenderness in the right iliac fossa (or where the appendix is lying). Biliary colic differs from appendicular, intestinal, or ureteric colic in that the pain builds up with intensity over a period of a few hours; it does not wax and wane.

Cardiac arrhythmias and pain are somehow linked to biliary disease, but the mechanism is obscure. Continuous electrocardiographic tracings during an operation may show an arrhythmia when traction is applied to the gall-bladder and the cystic duct. Many patients lose their cardiac symptoms after cholecystectomy.

Sir Zachary Cope, authority on the diagnostic problems of the acute abdomen which perplex the practitioner and surgeon, himself suffered recently from an attack of biliary colic and acute cholecystitis and has described it at p. 147. His trained senses recorded the early symptoms of the disease and his "cardiac link" symptoms. He makes a special point of the palpation of a tense, painless swelling-presumably the gall bladder-during the attack of colic. The swelling disappeared as the attack subsided, and he attributes it to a temporary obstruction of the cystic duct. Possibly this painless swelling has been often noted by practitioners who are at the bedside of their patient during an attack of colic. Certainly in hospital practice patients with acute biliary problems are often referred by their doctors with letters describing a palpable gall-bladder which is impalpable on arrival at hospital.

\section{Home from Hospital}

The Dan Mason Nursing Research Committee has issued its last report, ${ }^{1}$ which must be a matter of regret to anyone who values factual information on nursing and related topics. This research project studied the needs for home care of 533 people discharged from hospital and how far these needs had been met. They came from two areas; the one in the north was an industrial area with rural surroundings, and the other in the home counties had a range of socio-economic groups. The inquiry sought to find what patients themselves felt their needs to be, how far their expectations were fulfilled, and what were the existing arrangements and community care for people leaving hospital. Patients included in the sample were seen just before discharge and about two and ten weeks later at home.

The most fortunate ones (apart from those with no special needs returning to their families) were the maternity patients; $75 \%$ of them had been asked about their home arrangements by the medical social workers as against only $15 \%$ of the general patients. There is a good routine for notifying health visitors about new babies, and mothers who needed home helps usually got them, though it should be noticed that a sizeable number thought after a period at home that their discharge had been too early, and felt very tired.

It is a different matter when one reads about the old, the incontinent, the solitary, and those suffering from malignant disease. Fifty-two patients had multiple needs, and in these cases "life was often grim for the patient and his family." Thumbnail sketches are given of 18 patients for whom society offered little. A 90-year-old widow, blind, with untreated carcinoma of rectum, sat in bed or on the commode all day "waiting for the end to come." An epileptic who had attempted suicide and whose husband was in prison, returned to two rooms with five children, one mentally retarded, and with no support arranged. A 98-year-old hemiplegic, doubly incontinent, was sent home to a daughter and son-in-law, both over 75 , with no community services arranged.

One deplorable point is that 28 patients said they could not get adequate relief from pain, mostly because of doubt about who was prescribing for them. Five patients with malignant disease subsequently rang up the interviewer for advice; "all were desperate for effective analgesics, and could not think to whom to turn."

The main impression is of failure of communication. Hospitals know little of their patients' home circumstances, doctors do not hear of their patients' hospital treatment, patients do not know of the sources of help that are available. There is a great shortage of home helps, especially for those whose needs are greatest. Somehow the supply must be increased. The success of practice-attachment for district nurses and health visitors has been so great that perhaps home helps could be added to the team, and the morale-raising effect of working in a group might aid recruitment. Nobody in or outside hospital can feel complacent about the level of home care described in this report.

Skeet, M., Home from Hospital. London, Dan Mason Research Committec of the National Florence Nightingale Memorial Committee of Great Britain and Northern Ireland, 1970. 